\title{
Isolating Majorana fermions with finite Kitaev nanowires and temperature: the universality of the zero-bias conductance
}

\author{
V. L. Campo Jr ${ }^{1, *}$, L. S. Ricco ${ }^{2}$ and A. C. Seridonio ${ }^{2,3}$ \\ ${ }^{1}$ Departamento de Fúsica, Universidade Federal de São Carlos, Rodovia Washington Luiz, \\ km 235, Caixa Postal 676, 13565-905, São Carlos, São Paulo, Brazil \\ ${ }^{2}$ Departamento de Física e Química, Unesp - Univ Estadual Paulista, 15385-000, Ilha Solteira, São Paulo, Brazil \\ ${ }^{3}$ Instituto de Geociências e Ciências Exatas - IGCE, Universidade Estadual Paulista, \\ Departamento de Física, 13506-970, Rio Claro, São Paulo, Brazil and \\ *vlcampo@df.ufscar.br
}

\section{Abstract}

The zero-bias peak (ZBP) is understood as the definite signature of a Majorana bound state (MBS) when attached to a semi-infinite Kitaev nanowire (KNW) nearby zero temperature. However, such characteristics concerning the realization of the KNW are indeed elusive, since they constitute a profound experimental challenge. Against this scenario, we explore theoretically a QD connected to a topological KNW of finite size and show that at a non-zero temperature regime, the MBS become decoupled from each other if one tunes the system into the leaked Majorana fermion fixed point. Universal aspects of the temperature dependence of the zero-bias conductance are explored to offer additional signatures of the presence of MBS in the device. This work is a short version of our previously published work [1].

\section{Introduction}

After the advent and understanding of topological phases of matter, the proposal of topological quantum computation [2-4], which is protected against decoherence, including operations with isolated Majorana quasiparticle excitations, has triggered a remarkable theoretical and experimental sinergy in the condensed matter physics community [5-8]. Among the several theoretical proposals [9-16], the one-dimensional topological Kitaev nanowire (KNW), exibiting $p$-wave superconductivity feature [17] has been considered the paramount candidate to engineer isolated Majorana bound states (MBSs) at its ends.

The presence of the isolated MBSs at the edges of the KNW is inferred from tunneling spectroscopy, by analyzing the behavior of the zero-bias peak (ZBP) in the conductance profiles [18-20], which should be a hallmark of the MBS presence. This demands manufacturing long KNWs to prevent the MBSs overlapping and the consequent ZBP quenching at very low temperatures, what is considered a hard experimental challenge.

In this work, we explore the quantum dot (QD)-Kitaev nanowire (KNW) [21-26] hybrid setup sketched in Fig. 1. We address the interplay between thermal broadening and overlapped MBSs and found that effectively uncou- pled edge-MBSs can pop-up at relatively large temperature ranges.

We discuss the fixed points of the model and perform a numerical renormalization group analysis $[27,28]$ to study the crossovers between them and the temperature dependence of the conductance. Of central importance is the leaked Majorana fixed point, that we find to occur in the vicinity of a characteristic temperature that depends solely on the KNW properties, although such a vicinity has a width that depends on the whole set of model parameters. The leakage of the Majorana fermion was first predicted theoretically in Ref. [23] and has been reported experimentally by Deng et al [20]. Further, we find rigorously the crossover temperatures and derive an analytic expression describing the universal behavior of the zero-bias conductance along the crossovers. The universal behavior reveals a more complete signature of the physical system.

\section{Methods and Results}

Assuming that the Zeeman spliting in the QD is large enough so that we can neglect the transport of spin down electrons through it, we consider the effective model with 


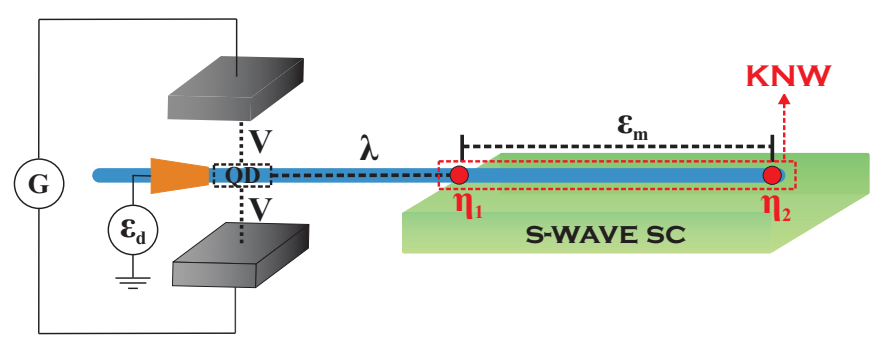

Figure 1. Sketch of the setup here explored. A piece of semiconductor nanowire is placed on a s-wave superconductor (SC) material. In this semiconductor-superconductor segment, by considering suitable Zeeman field and spin-orbit coupling, a topological KNW emerges, giving rise to overlapped $\left(\varepsilon_{m}\right)$ MBSs $\eta_{1}$ and $\eta_{2}$ at their edges. A QD (gate tunable energy $\epsilon_{d}$ ) between two metallic leads (V coupling) is built-in the semiconducting segment, where $\eta_{1}$ leaks into ( $\lambda$ coupling) and can be detected as a ZBP.

spinless fermions [21, 26], whose Hamiltonian is given by

$$
\begin{aligned}
H & =\sum_{k, \alpha=U, L} \epsilon_{k} c_{k, \alpha}^{\dagger} c_{k, \alpha}+V \sum_{k, \alpha=U, L}\left(c_{k, \alpha}^{\dagger} d+d^{\dagger} c_{k, \alpha}\right) \\
& +\epsilon_{d} d^{\dagger} d+i \epsilon_{m} \eta_{1} \eta_{2}+\sqrt{2} \lambda\left(d-d^{\dagger}\right) \eta_{1},
\end{aligned}
$$

where the first term describes the conduction electrons in the upper (U) and lower (L) leads. We assume halffilled conduction bands in the particle-hole symmetric regime, with a constant density of states equal to $\rho$, $-D \leq \epsilon_{k, \alpha} \leq D$ and Fermi energy equal to zero. The QD here has only one energy state $\epsilon_{d}$ that is hybridized with the conduction states in the leads through the second term in the Hamiltonian, resulting in a linewidth $\Gamma=\pi \rho V^{2}$. We assume here symmetric coupling to the leads. The $\mathrm{KNW}$ is assumed to be in the topological phase with two MBSs at its ends $\left(\eta_{i}=\eta_{i}^{\dagger}, \eta_{i} \eta_{i}=1 / 2\right)$, with an overlap amplitude $\epsilon_{m} \sim e^{-L / \xi}$ between them, where $L$ is the length of the KNW and $\xi$ is the superconductor coherence length. The last term in the Hamiltonian represents the coupling between the MBS $\eta_{1}$ and the QD single state.

We consider now even and odd conduction states, $e_{k}=$ $\left(c_{k, U}+c_{k, L}\right) / \sqrt{2}$ and $o_{k}=\left(c_{k, U}-c_{k, L}\right) / \sqrt{2}$ and also the nonlocal fermionic operators $b=\left(\eta_{1}+i \eta_{2}\right) / \sqrt{2}$ and $b^{\dagger}=\left(\eta_{1}-i \eta_{2}\right) / \sqrt{2}\left(\left\{b, b^{\dagger}\right\}=1,\{b, b\}=0\right)$, to rewrite the model Hamiltonian as

$$
\begin{aligned}
H & =\sum_{k} \epsilon_{k}\left(e_{k}^{\dagger} e_{k}+o_{k}^{\dagger} o_{k}\right)+\sqrt{2} V \sum_{k}\left(e_{k}^{\dagger} d+d^{\dagger} e_{k}\right) \\
& +\epsilon_{d} d^{\dagger} d+\epsilon_{m}\left(b^{\dagger} b-\frac{1}{2}\right)-\lambda\left(d^{\dagger} b+b^{\dagger} d\right) \\
& -\lambda\left(d^{\dagger} b^{\dagger}+b d\right),
\end{aligned}
$$

where the odd conduction states are decoupled from the $\mathrm{QD}$ and the number of fermions is not conserved.
The zero-bias conductance as a function of the temperature $T$ can be calculated from[27]

$$
G(T)=\frac{2 e^{2}}{h} \pi \Gamma\left[\frac{1}{k_{B} T} \frac{1}{Z} \sum_{n, m} \frac{|\langle n|d| m\rangle|^{2}}{e^{\beta E_{n}}+e^{\beta E_{m}}}\right]
$$

where $Z$ stands for the partition function for the even Hamiltonian in Eq. (2), wherein the labels $n$ and $m$ run over their eigenstates, whose energies are $E_{n}$ and $E_{m}$ respectively. Alternatively, we can rewrite Eq. (3) as [29]

$$
G(T)=\frac{2 e^{2}}{h} \Gamma \int_{-\infty}^{\infty} \operatorname{Im}\left\{G_{d, d}(\omega)\right\}\left(\frac{\partial f}{\partial \omega}\right) d \omega
$$

where $f(\omega)$ is the Fermi-Dirac function. The QD Green's function can be promptly obtained [1] from the equation of motion [29] procedure.

Although the model system is quadratic and the quantum dot Green functions can be obtained analytically, we have used the Numerical Renormalization Group (NRG) method [30-33] to compute the conductance as a function of the temperature [27, 28], since this method allows one to follow in a natural way the crossovers between fixed points. We refer the reader to the literature for a detailed account of the method.

For a vanishing coupling $\lambda$ between the QD and the KNW or for $\epsilon_{m} \rightarrow \infty$, so that the fermionic state $b$ becomes empty, we end up with a simple resonant level model. For non-vanishing coupling $\lambda$ and $\epsilon_{m}=0$ (infinite KNW), we can find [1] that the zero-bias conductance through the QD approaches $0.5 e^{2} / h$ at $\omega=0$ when the temperature $T \rightarrow 0$, whatever the values of $\epsilon_{d}$ and $\Gamma$, being a signature of the leakage of the MBS $\eta_{1}$ into the $\mathrm{QD}[23]$. However, any nonzero $\epsilon_{m}$ makes the conductance change to its resonant level model value

$$
G_{0}=\frac{e^{2}}{h} \frac{4 \Gamma^{2}}{\epsilon_{d}^{2}+4 \Gamma^{2}}=\frac{e^{2}}{h} \sin ^{2}(\delta)
$$

in the limit of zero temperature, where $\delta$ is the phaseshift at the Fermi level. This fact prompt us to a more detailed investigation of the temperature dependence of the conductance.

Fixed points - Fig. 2 is clarifying. At high temperatures, when we can effectively consider both $\lambda$ and $\epsilon_{m}$ equal to zero, the conductance approaches $G_{0} \approx$ $0.09 e^{2} / h$ and the system is close to the free Majorana fermions fixed point. Then, as the temperature is lowered, the coupling finally emerges, leading to a crossover from a conductance equal to $G_{0}$ towards a conductance equal to $0.5 e^{2} / h$, when the system approaches the leaked Majorana fixed point. This value remains stable in a certain temperature range. At some point, however, the tiny coupling $\left(\epsilon_{m}\right)$ between the Majorana fermions emerges and they become strongly coupled, yielding a new crossover ending with $G_{0}$ recovered and the system in the strongly coupled Majorana fermions. For a very long $\mathrm{KNW}\left(\epsilon_{m} \rightarrow 0\right)$, the last crossover will be shifted 


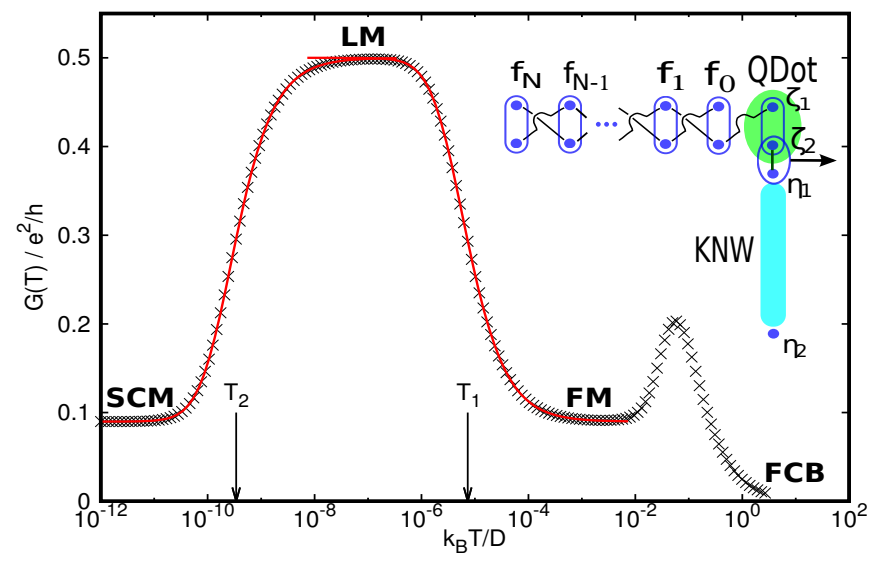

Figure 2. Zero-bias conductance as function of temperature in logarithm scale for $\epsilon_{d}=0.1 D, \Gamma=0.005 \pi D, \lambda=0.0008 D$, $\epsilon_{m}=5 \times 10^{-8} D$. Continuous lines are given by Eqs. (11) and (12)with crossover temperatures $T_{1}$ (Eq. (8)) and $T_{2}$ (Eq. (9)). The inset pictorially shows the Majorana representation of the problem in the leaked Majorana (LM) fixed point, considering the NRG tight-binding description of the conduction band, wherein each site comprises two Majorana fermions denoted by blue circles. Majorana fermions $\eta_{1}$ and $\zeta_{2}$ are strongly coupled and removed from the system (see the horizontal arrow). The Majorana fermion $\zeta_{1}$ is coupled to one chain of Majorana fermions in the conduction band, giving rise to half of the single-particle excitations of the $\epsilon_{d}=0$ resonant level model. The other chain gives rise to half of the singleparticle excitations of the free conduction band, that do not contribute to the conductance. This implies a conductance equal to $0.5 e^{2} / h$.

to very low temperatures, allowing the observation of an essentially stable conductance value equal to $0.5 e^{2} / h$. More relevant than particular values of some physical properties in the $T \rightarrow 0$ limit is the universal behavior of these physical properties during the crossover as some parameter is changed, typically the temperature. Below, we briefly discuss the fixed points and then proceed to the analysis of the universal behavior in the temperature dependence of the conductance.

Free conduction band (FCB) fixed point: This corresponds to do $V, \epsilon_{d}, \lambda, \epsilon_{m} \rightarrow 0$ in the model Hamiltonian. Both QD level and nonlocal fermionic level $b$ are detached from the conduction band and can be empty or occupied, so that any energy is fourfold degenerate. The excitations are those in a free conduction band. The system would be close to this fixed point at temperatures $k_{B} T \gg \Gamma, \epsilon_{d}, \lambda, \epsilon_{m}$, where the conductance goes to zero. Since the temperature must necessarily be lower than the effective superconducting gap in the KNW, this fixed point will not be observed in general.

Free Majorana fermion (FM) fixed point: In this case, $\lambda, \epsilon_{m} \rightarrow 0$ together with the emulation of the resonant level model in the limit $k_{B} T \ll \Gamma$. The energies are twofold degenerate, since the fermionic level can be empty or occupied. The excitations in the conduction band have a phase-shift $\delta$, with $\cot \delta=\epsilon_{d} / 2 \Gamma$.
Strongly coupled Majorana fermions (SCM) fixed point: Here, we regard $\epsilon_{m} \rightarrow \infty$. The MBSs become strongly coupled and the nonlocal fermionic level $b$ remains empty. The system becomes the resonant level model, with the same conductance and same excitations as in the FM fixed point, but without degeneracy.

Leaked Majorana fermion (LM) fixed point: This corresponds to do $\lambda \rightarrow \infty$ in the model Hamiltonian. From Eq. (1), the MBSs $\eta_{1}$ and $\zeta_{2}=i\left(d^{\dagger}-d\right) / \sqrt{2}$ become infinitely coupled, leading to a nonlocal fermionic level with infinite energy, that remains empty. But, we still have the MBS $\zeta_{1}=\left(d^{\dagger}+d\right) / \sqrt{2}$ in the QD, so that the leaked Majorana fixed point is described by the following Hamiltonian:

$$
H_{L M}=\sum_{k} \epsilon_{k} e_{k}^{\dagger} e_{k}+V \sum_{k}\left(e_{k}^{\dagger} \zeta_{1}+\zeta_{1} e_{k}\right)
$$

Essentially, the MBS has leaked from the KNW edge into the QD [20, 23]. However, it is coupled to the even conduction states so that this leaking process will reach the conduction band.

With the MBS $\zeta_{1}$ at the QD level and $\eta_{2}$ at the other far edge of the KNW, we introduce a new fermionic operator, $a=\left(\zeta_{1}+i \eta_{2}\right) / \sqrt{2}$, to rewrite $H_{L M}$ as

$$
H_{L M}=\sum_{k} \epsilon_{k} e_{k}^{\dagger} e_{k}+\frac{V}{\sqrt{2}} \sum_{k}\left(e_{k}^{\dagger} a+e^{\dagger} a^{\dagger}+\text { h.c. }\right) \text {. }
$$

As indicated in the caption of Fig. 2 and explained in detail in Ref. [1], one half of the single-particle excitations of $H_{L M}$ are of free-conduction band type and another half of them are of $\epsilon_{d}=0$ resonant level model type. Only the last set of excitations can contribute to the conductance, leading to the characteristic value of $0.5 e^{2} / h$ as $T \rightarrow 0$.

Now we turn to the problem of carefully identifying universal behavior in the zero-bias conductance. In Fig. 3a, we show the conductance for different sets of model parameters. We have used $\epsilon_{d}=0$ and $\Gamma=$ $\pi 0.005 D$, changing $\epsilon_{m}$ and $\lambda$. Therefore, we have conductance $G_{0}=1.0 e^{2} / h$ in the FM and SCM fixed points. It is clear from Fig. 3a that the crossovers occur around parameter-dependent temperatures $T_{1}$ (from the FM fixed point to the LM fixed point by lowering the temperature) and $T_{2}$ (from the LM fixed point to the SCM fixed point). We have found from the numerical results that

$$
k_{B} T_{1}=\frac{2}{\Gamma} \frac{\lambda^{2}}{\left[1+\left(\frac{\epsilon_{d}}{2 \Gamma}\right)^{2}\right]},
$$

and

$$
k_{B} T_{2}=\frac{\Gamma}{2}\left[1+\left(\frac{\epsilon_{d}}{2 \Gamma}\right)^{2}\right]\left(\frac{\epsilon_{m}}{\lambda}\right)^{2} .
$$

One could expect that the crossover to the SCM fixed point would happen when $k_{B} T$ becomes of order $\epsilon_{m}$ since this is the energy scale of the coupling between the origi- 

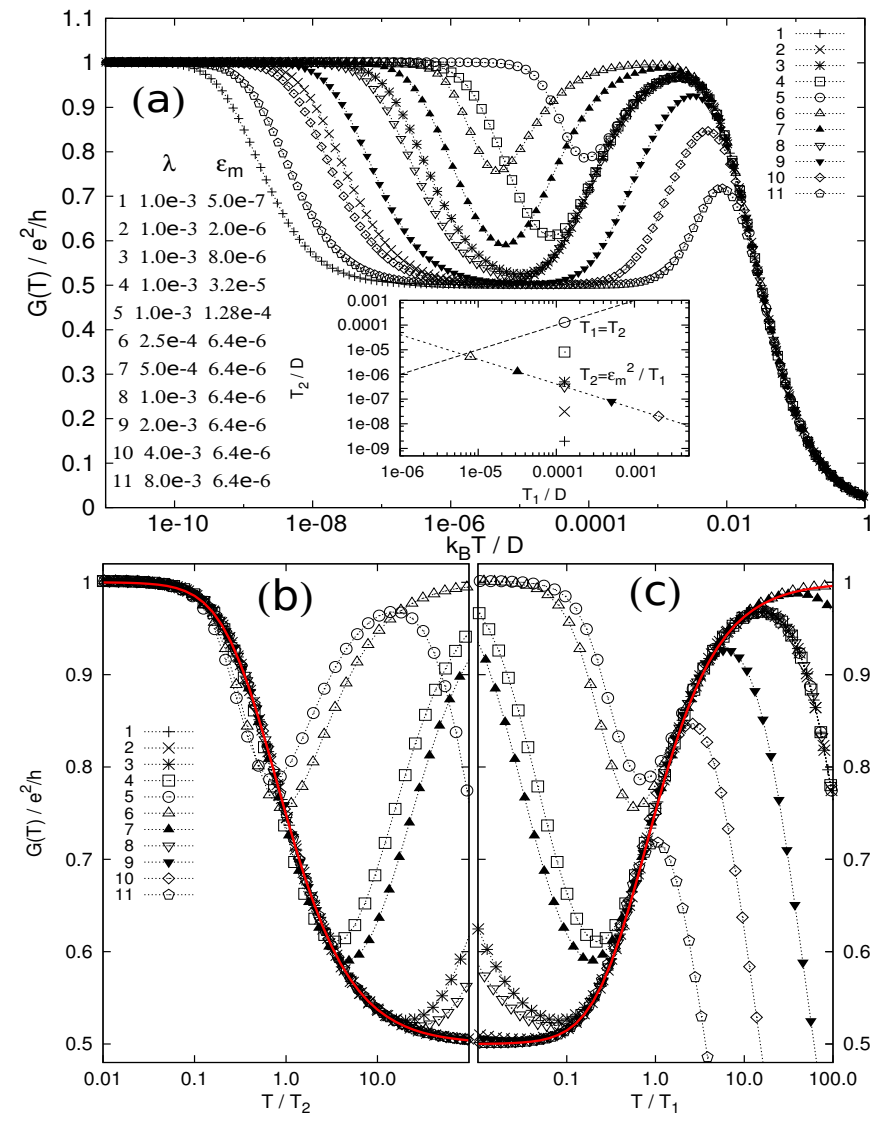

Figure 3. (a) Zero-bias conductance as function of temperature in logarithm scale for 11 different model parameter pairs $\left(\lambda, \epsilon_{m}\right)$. In all curves, $\epsilon_{d}=0$ and $\Gamma=\pi 0.005 D$. The inset shows the distribution of the corresponding crossover temperatures $T_{1}$ and $T_{2}$ (Eqs. (8) and (9)). Curves 1-5 have the same $\lambda$ and the same $T_{1}$. Curves $6-11$ have the same $\epsilon_{m}$ and their minima between the crossovers are approximately at the same point (Eq. (10)). (b) and (c) Conductance as a function of $T / T_{2}$ and $T / T_{1}$, respectively. The points collapse into the continuous lines in Eq. (11) with $\mathcal{H}(t)$ in Eq. (12). In (b), when $T_{1}$ is not much higher than $T_{2}$, deviations from the universal curve will start at low temperatures. In (c), deviations at $T<T_{1}$ occur when $T_{2}$ is not much lower than $T_{1}$ while deviations at $T>T_{1}$ occur when $T_{1}$ is not much smaller than $\Gamma / k_{B}$.

nal MBSs $\eta_{1}$ and $\eta_{2}$. We have found, however, that such a crossover happens when $T \sim T_{2}$ instead. This difference can be understood because the coupling is not between the original MBSs but between $\eta_{2}$ and a Majorana fermion that has leaked to the QD and to the conduction band as discussed above.

From Eqs. (8) and (9), we have

$$
\sqrt{T_{1} T_{2}}=\epsilon_{m} / k_{B}
$$

that means the plateau at $0.5 e^{2} / h$ in the conductance will be centered at $T \sim \epsilon_{m} / k_{B}$, being well defined only if $T_{2} \ll \epsilon_{m} / k_{B} \ll T_{1}$.

Scaling the temperature by $T_{2}\left(T_{1}\right)$, the crossover por- tions around $T_{2}\left(T_{1}\right)$ of the different curves in Fig. 3a collapse into the same curve as shown in Fig. 3b(c). This universal curve is given by Eqs. (11) and (12) below. From Figs. 3b and 3c, we see that the temperature must be changed by at least two orders of magnitude to complete the crossover, what demands $T_{1}>100 T_{2}$ to clearly have the system in the LM fixed point. The ratio $T_{1} / T_{2}=\left(k_{B} T_{1} / \epsilon_{m}\right)^{2}$ depends on all model parameters, what can help to tune it large enough. Naturally, the limit $T_{1} \gg T_{2}$ can be achieved by considering very long KNWs, i.e., $\epsilon_{m} \rightarrow 0$, once $T_{1}$ is independent of $\epsilon_{m}$.

In general, we expect $[27,28]$ that the conductance between a low-temperature fixed-point where $G=G_{l}$ and a high-temperature fixed-point where $G=G_{h}$ be given by

$$
G=\frac{G_{l}+G_{h}}{2}+\frac{G_{l}-G_{h}}{2} \mathcal{H}\left(T / T^{\star}\right),
$$

where $\mathcal{H}\left(t=T / T^{\star}\right)$ is a universal function characteristic of the crossover and $T^{\star}$ is the crossover temperature. For the model system studied here, we have found that the same universal function describes the crossover between SCM and LM fixed points and between LM and FM fixed points, being given by [1]

$$
\mathcal{H}(t)=\int_{-\infty}^{\infty}\left[\frac{1-u^{2} t^{2}}{1+u^{2} t^{2}}\right] \frac{e^{u}}{\left(e^{u}+1\right)^{2}} d u
$$

\section{Discussion}

To make contact with a possible experimental realization of the setup in Fig. 1, we start by taking a QD energy $\epsilon_{d}=0$. To fix the other model parameters, we set $T_{1} / T_{2}=100$ and $\Gamma / k_{B} T_{1}=10$ to separate the crossovers. From Eqs. (8) and (9), we have $\frac{\epsilon_{m}}{\lambda}=\frac{0.1}{\sqrt{5}}$ and $\frac{\lambda}{\Gamma}=\frac{0.5}{\sqrt{5}}$.

We now consider a Kitaev nanowire long enough so that $\epsilon_{m}=0.001 \mathrm{meV}$. This can be achieved experimentally [34] for a InAs nanowire of $1500 \mathrm{\eta m}$. Therefore, we get $\lambda=0.02236 \mathrm{meV}$ (very close to the reported value of $0.02353 \mathrm{meV}[26])$ and $\Gamma=0.1 \mathrm{meV}$. This value of $\Gamma$ is $5 \times$ larger than what is reported in Refs. 23 and 26, for example. However, since the barrier between the QD and the leads can be gate-tunable, $\Gamma$ can be adjusted. To ensure the spinless model validity, we must have a Zeeman spliting in the QD large compared to $\Gamma$. For a InSb nanowire, $g \mu_{B} B$ is of order $0.8 \mathrm{meV}$ [26] for relatively small magnetic fields $(\sim 300 \mathrm{mT})$. Therefore, for the spin up energy $\epsilon_{d}=0$, we have the spin down energy around $8 \Gamma$ s above the Fermi level, what makes negligible the contribution of the spin down channel to the conductance. For the half bandwidth $D$, we follow previous works $[23,26,35]$ and adopt $D=20 \mathrm{meV} \gg \Gamma$.

In Fig. 4a, we show the conductance calculated for five different values of $\epsilon_{d}$, with the remaining model parameters fixed with the values determined above. For $\epsilon_{d}=0$ we have, by construction, $T_{1} / T_{2}=100$, with the system close to the LM fixed point and the conductance close to 

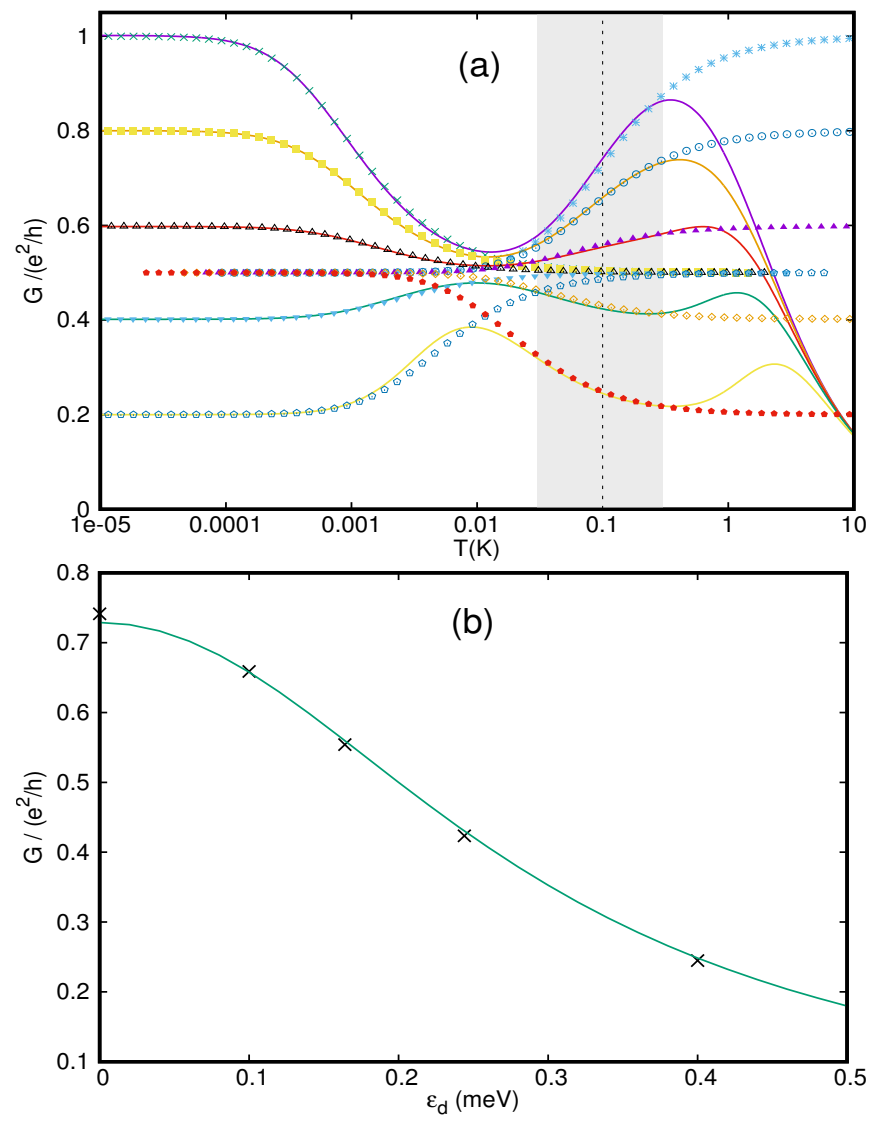

Figure 4. (a) Conductance as a function of the temperature for different values of the QD energy. Experimentally accessible values were used for the other model parameters (see main text). From top to bottom, we have $\epsilon_{d}=0.0,0.1$, $0.164,0.244$ and $0.4 \mathrm{meV}$, respectively. The continuous lines represent the calculated conductances for each $\epsilon_{d}$, while the points represent the universal expression [Eq.(11)] for both $T^{\star}=T_{1}$ and $T^{\star}=T_{2}$ with $\mathcal{H}(t)$ given by Eq. (12). In the highlighted range $(0.03 \mathrm{~K}<\mathrm{T}<0.3 \mathrm{~K})$, the universal expression (with $T^{\star}=T_{1}$ ) describes accurately the conductance behavior. This also happens for $T \sim T_{2}$, but, in this case, the temperatures are more difficult to be achieved experimentally. (b) The continuous line represents the conductance calculated from Eq.(11) at $T=0.1 \mathrm{~K}$ and the points are the exactly computed conductance at this temperature for the values of $\epsilon_{d}$ used in panel (a).
$0.5 e^{2} / h$ when $k_{B} T \sim \epsilon_{m}$. For $\epsilon_{d}=0.4 \mathrm{meV}$, we have $T_{1} / T_{2}=4$, and the conductance is never close to $0.5 e^{2} / \mathrm{h}$. However, there are some ranges of temperature around $T_{1}$ and $T_{2}$ where the conductance is well described by the universal expression of Eq. (11). For a given temperature $T$ close to $T_{1}$, we can accurately compute the conductance as a function of $\epsilon_{d}$ from Eq. (11) $\left(G_{l}=0.5 e^{2} / h\right.$, $G_{h}=G_{0}\left(\epsilon_{d}\right), T^{\star}=T_{1}\left(\epsilon_{d}\right)$ and $\mathcal{H}(t)$ from Eq. (12)) as can be seen in Fig. $4 \mathrm{~b}$, where $T=0.1 \mathrm{~K}$. Even though the plateau in the conductance at $0.5 e^{2} / h$ was not observed in Fig. 4a, the behavior in Fig. 4b follows directly from the universal description of the crossover between the LM and the FM fixed points, being another possible signature of the MBS in the system.

In summary, we have determined the universal behavior of the zero-bias conductance for the simple spinless model in Eq. (1) along the crossovers connected to the LM fixed point. This enlarges the signature of the MBS in the end of the KNW and can help to reveal its presence when the LM fixed point is not fully achieved. Even with a finite KNW, it may be possible to set up the model parameters to have $T_{1} \gg T_{2}$ and a reasonably large temperature range with conductance close to $0.5 e^{2} / h$.

\section{Acknowledgments}

We thank the funding Brazilian agencies $\mathrm{CNPq}$ (307573/2015-0)), CAPES and São Paulo Research Foundation (FAPESP) - grant: 2015/23539-8.
[1] V. L. Campo, L. S. Ricco, and A. C. Seridonio, Phys. Rev. B 96, 045135 (2017).

[2] A. Y. Kitaev, Annals of Physics 303, 2 (2003).

[3] S. D. Sarma, M. Freedman, and C. Nayak, npj Quantum Information 1, 15001 (2015).

[4] C. Nayak, S. H. Simon, A. Stern, M. Freedman, and S. D. Sarma, Reviews of Modern Physics 80, 1083 (2008).

[5] J. Alicea, Y. Oreg, G. Refael, F. Von Oppen, and M. P. Fisher, Nature Physics 7, 412 (2011).

[6] M. Leijnse and K. Flensberg, Semiconductor Science and Technology 27, 124003 (2012).

[7] J. Alicea, Reports on Progress in Physics 75, 076501
(2012)

[8] C. Beenakker, Annu. Rev. Condens. Matter Phys. 4, 113 (2013).

[9] L. Fu and C. L. Kane, Phys. Rev. Lett. 100, 096407 (2008).

[10] J. D. Sau, R. M. Lutchyn, S. Tewari, and S. Das Sarma, Phys. Rev. Lett. 104, 040502 (2010).

[11] J. Alicea, Phys. Rev. B 81, 125318 (2010).

[12] R. M. Lutchyn, J. D. Sau, and S. Das Sarma, Phys. Rev. Lett. 105, 077001 (2010).

[13] Y. Oreg, G. Refael, and F. von Oppen, Phys. Rev. Lett. 105, 177002 (2010). 
[14] J. Linder, Y. Tanaka, T. Yokoyama, A. Sudbø, and N. Nagaosa, Phys. Rev. Lett. 104, 067001 (2010).

[15] A. Cook and M. Franz, Phys. Rev. B 84, 201105 (2011).

[16] S. Nadj-Perge, I. K. Drozdov, B. A. Bernevig, and A. Yazdani, Phys. Rev. B 88, 020407 (2013).

[17] A. Y. Kitaev, Physics-Uspekhi 44, 131 (2001).

[18] V. Mourik, K. Zuo, S. M. Frolov, S. Plissard, E. Bakkers, and L. Kouwenhoven, Science 336, 1003 (2012).

[19] S. Nadj-Perge, I. K. Drozdov, J. Li, H. Chen, S. Jeon, J. Seo, A. H. MacDonald, B. A. Bernevig, and A. Yazdani, Science 346, 602 (2014).

[20] M. T. Deng, S. Vaitiekenas, E. B. Hansen, J. Danon, M. Leijnse, K. Flensberg, J. Nygård, P. Krogstrup, and C. M. Marcus, Science 354, 1557 (2016).

[21] D. E. Liu and H. U. Baranger, Physical Review B 84, 201308 (2011).

[22] Y. Cao, P. Wang, G. Xiong, M. Gong, and X.-Q. Li, Physical Review B 86, 115311 (2012).

[23] E. Vernek, P. Penteado, A. Seridonio, and J. C. Egues, Physical Review B 89, 165314 (2014).

[24] D. E. Liu, M. Cheng, and R. M. Lutchyn, Physical Review B 91, 081405 (2015).

[25] Z.-Z. Li, C.-H. Lam, and J. You, Scientific reports 5
$(2015)$

[26] D. A. Ruiz-Tijerina, E. Vernek, L. G. D. da Silva, and J. Egues, Physical Review B 91, 115435 (2015).

[27] M. Yoshida, A. C. Seridonio, and L. N. Oliveira, Phys. Rev. B 80, 235317 (2009).

[28] A. C. Seridonio, M. Yoshida, and L. N. Oliveira, Phys. Rev. B 80, 235318 (2009).

[29] J. A.-P. Hartmut Haug, Quantum Kinetics in Transport and Optics of Semiconductors, Vol. 123 (Springer-Verlag Berlin Heidelberg, 2008).

[30] K. G. Wilson, Rev. Mod. Phys. 47, 773 (1975).

[31] H. R. Krishna-murthy, J. W. Wilkins, and K. G. Wilson, Phys. Rev. B 21, 1003 (1980).

[32] H. R. Krishna-murthy, J. W. Wilkins, and K. G. Wilson, Phys. Rev. B 21, 1044 (1980).

[33] R. Bulla, T. A. Costi, and T. Pruschke, Rev. Mod. Phys. 80, 395 (2008).

[34] S. M. Albrecht, A. Higginbotham, M. Madsen, F. Kuemmeth, T. S. Jespersen, J. Nygård, P. Krogstrup, and C. Marcus, Nature 531, 206 (2016).

[35] D. Rainis, L. Trifunovic, J. Klinovaja, and D. Loss, Phys. Rev. B 87, 024515 (2013). 